\title{
Concentrated Growth Factor, Platelet-rich Fibrin, Mineral Trioxide Aggregate as Direct Pulp-capping Agents: Case Reports
}

\author{
AM Swathi $^{1}$, Mahendran Kavitha ${ }^{2}$
}

\begin{abstract}
Background: Direct pulp capping is a procedure in which the exposed vital pulp is covered with a protective dressing or a base placed directly over the site of exposure in an attempt to preserve the pulp vitality. The most visible reparative response to pulp exposure is the deposition of reparative dentin, which is affected by pulp-capping material. Recently, platelet concentrates like concentrated growth factor (CGF) and platelet-rich fibrin (PRF) are being used in various fields in dentistry due to their regenerative properties. They can be used as pulp-capping agents, as the growth factors present in these platelet concentrates can stimulate dental pulp cells and stimulate odontoblastic differentiation, leading to reparative dentin formation.

Case description: Direct pulp capping was carried out in mandibular molars, with radiographic evidence of deep caries approximating pulp chamber and with no evidence of periapical pathology. The pulp exposure of $0.5-1 \mathrm{~mm}$ was capped with CGF/PRF/mineral trioxide aggregate (MTA), over which glass ionomer cement (GIC) liner was placed and final restoration was performed with light cure composite (Tetric N Ceram, Ivoclar Vivadent). Patients were evaluated clinically after 3 months, to check for pulp vitality using cold and electric pulp testing, periodontal probing depth, mobility, pain, and tenderness to percussion. Cone-vbeam computed tomography (CBCT) evaluation was carried out after 3 months, to assess dentin bridge formation.

Conclusion: Platelet-rich fibrin and CGF were found to be promising pulp-capping agents and their clinical efficacy was comparable to MTA. Clinical significance: The promising results of platelet concentrates as direct pulp-capping agents opens a new avenue for vital pulp therapy procedures.
\end{abstract}

Keywords: Concentrated growth factor, Direct pulp capping, Mineral trioxide aggregate, Platelet-rich fibrin.

Journal of Operative Dentistry and Endodontics (2020): 10.5005/jp-journals-10047-0087

\section{INTRODUCTION}

The vitality of the pulp is essential for the longevity of the tooth as pulp is the only living tissue of the tooth which nourishes, aids in reparative tissue formation, and also exhibits immune functions. Direct pulp capping is the treatment of an exposed vital pulp by sealing the pulpal wound with a dental material placed directly on an iatrogenic or traumatic exposure to initiate the formation of reparative dentin and maintenance of the vital pulp. ${ }^{1}$

The search for an ideal pulp-capping material still continues to exist. Traditionally, calcium hydroxide formulations were used as pulpcapping agent and were once considered the gold standard for pulpcapping material. However, the material had several drawbacks like poor sealing ability with dentin, lack of ability to promote odontoblast differentiation in a consistent manner, was found to be cytotoxic in cell cultures and the reparative dentin formation induced by calcium hydroxide had tunnel defects. ${ }^{2}$ Mineral trioxide aggregate (MTA) is a bioactive, Portland cement-based, tricalcium silicate cement that has been shown to be a successful pulp-capping agent based on recent in vitro, animal, and in vivo studies. The material is non-absorbable, sets in the presence of moisture, has a relatively high compressive strength, and has a sustained high alkaline $\mathrm{pH} .{ }^{3}$ However, it poses drawbacks like its potency to cause tooth discoloration, presence of toxic elements in its composition, difficult handling properties, long setting time, cost ineffectiveness, the absence of a known solvent to retrieve the material, and the difficulty faced during of its removal post-hardening.

Recently, platelet concentrates like concentrated growth factor (CGF) and platelet-rich fibrin (PRF) are being used in various fields in dentistry due to their regenerative properties. They can

\begin{abstract}
1,2Department of Conservative Dentistry and Endodontics, Tamil Nadu Government Dental College and Hospital, The Tamil Nadu Dr MGR Medical University, Chennai, Tamil Nadu, India

Corresponding Author: AM Swathi, Department of Conservative Dentistry and Endodontics, Tamil Nadu Government Dental College and Hospital, The Tamil Nadu Dr MGR Medical University, Chennai, Tamil Nadu, India, Phone: +91 7397394109, e-mail: swathi1592@ gmail.com

How to cite this article: Swathi AM, Kavitha M. Concentrated Growth Factor, Platelet-rich Fibrin, Mineral Trioxide Aggregate as Direct Pulpcapping Agents: Case Reports. J Oper Dent Endod 2020;5(1):39-44.

Source of support: Nil

Conflict of interest: None
\end{abstract}

be used as pulp-capping agents, as the growth factors present in these platelet concentrates are known to play a crucial role in hard and soft tissue repair mechanisms, by exhibiting chemotactic and mitogenic properties that promote and modulate cellular functions. They can stimulate dental pulp cells into odontoblastic like cells, leading to reparative dentin formation. ${ }^{4}$

Following are three case reports on direct pulp capping using MTA, PRF, and CGF.

\section{Case Descriptions}

\section{Case 1}

History

A 27-year-old male patient reported to the department with a chief complaint of pain in right lower back teeth for the past 1 month. 
He gave a history of sharp pain, which was stimulated by cold food, which lasted for a few seconds and subsided on the cessation of stimuli.

\section{Clinical Examination}

Intraoral examination revealed deep dentinal caries involving buccal and occlusal surfaces in 46 (Fig. 1A). The tooth was not tender on percussion.

\section{Investigations}

Vitality testing using electric pulp test (EPT) and cold test (Endo-Ice, COLTENE) showed a positive response in 46.

Radiographic findings revealed radiolucency in the occlusal aspect approximating pulp in relation to 46 , with no evidence of thickening/widening of periodontal ligament (Fig. 1B).

\section{Provisional Diagnosis}

Reversible pulpitis in 46.

\section{Treatment Plan}

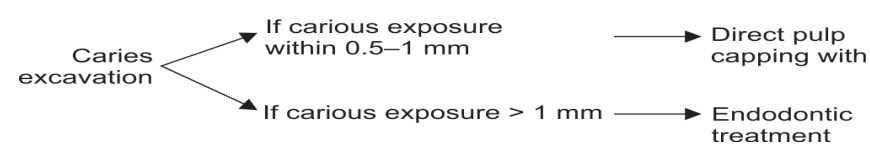

\section{Clinical Procedure}

Direct pulp-capping procedure: Oral prophylaxis was performed prior to the commencement of the treatment. Patients were given a prophylactic oral rinse of $0.2 \%$ chlorhexidine (Rexidine mouth rinse, ICPA products) just before the procedure. Under local anesthesia, lignocaine with 1:80,000 adrenaline (Lignox 2\%,
Warren, Indoco Remedies Ltd., India) and rubber dam isolation, caries detector dye (Prime Dental Products Pvt Ltd., India) was applied to the tooth for 30 seconds and rinsed to disclose the infected dentin. Caries excavation was performed using sterile diamond points [Round diamond bur BR 41/Straight fissure bur (SF 11 Mani Dia-Burs Inc.)] and high speed airotor handpiece (NSK, Tokyo, Japan) (Fig. 1C). Complete caries removal was ensured with caries indicator. Once there was a carious pulp exposure of $0.5-1$ $\mathrm{mm}$ (Fig. 1D), hemostatis was achieved by placing a sterile cotton soaked in $3 \% \mathrm{NaOCl}$. The cavities were then examined visually for arrest of bleeding.

CGF preparation: Concentrated growth factor was prepared just before its placement in the cavity as a pulp-capping agent. Nine milliliters of venous blood were drawn from the patient's antecubital vein and transferred into a sterile Vacuette test tube (Greiner Bio-One, Kremsmunster, Austria) without an anticoagulant (Fig. 1E). These tubes were immediately centrifuged in the CGF centrifugation machine (Medifuge, Silfradent, Italy) (Fig. 1F) using a variable rpm as follows: 30 seconds acceleration, 2 minutes at 2,700 rpm, 4 minutes at 2,400 rpm, 4 minutes at 2,700 rpm, 3 minutes at 3,000 rpm, and 36 seconds deceleration. At the end of the process, three blood fractions were identified: (1) Upper layer, platelet-poor plasma (PPP), which represents the liquid phase of plasma, (2) middle layer, representing the solid CGF, (3) lower layer, red blood cells (Fig. 1G). Concentrated growth factor was separated from red corpuscles base (preserving a small RBC layer) using sterile tweezers just after removal of PPP and then transferred into a sterile dappen dish (Fig. 1H). The CGF clot was compressed gently between moist sterile cotton gauze to remove the serum and to procure the CGF membrane (Fig. 11). The CGF
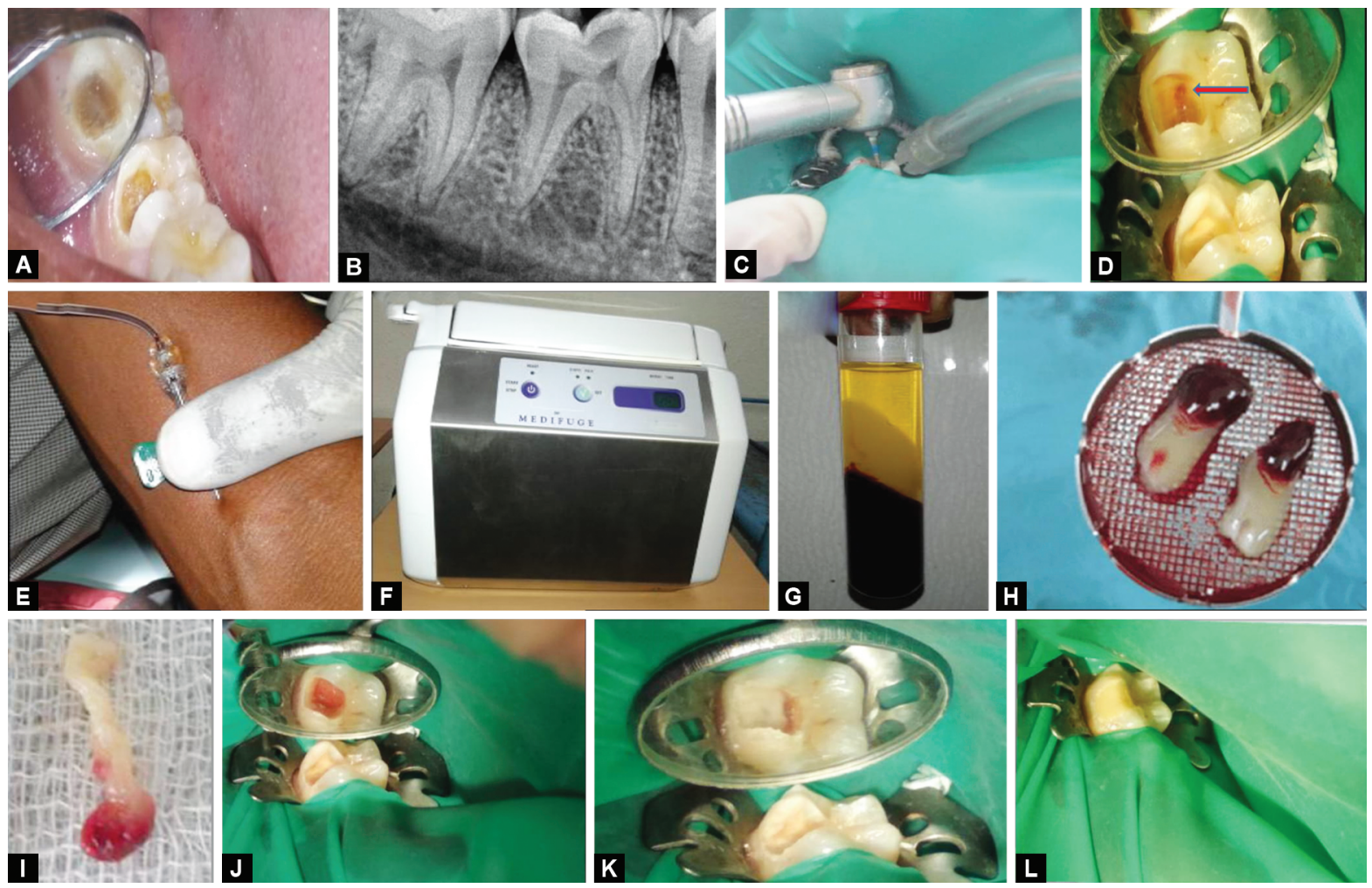

Figs 1 A to L: (A) Preoperative image; (B) Preoperative radiograph; (C) Caries excavation; (D) Pinpoint exposure; (E-H) CGF preparation in Medifuge (Silfradent, Italy); (I) CGF membrane; (J) placement of CGF; (K) GIC liner; (L) Light cure composite restoration 
membrane was then carried to the exposure and positioned with the tine of the sterile explorer (Fig. 1J).

Restorative procedure: After placement of the CGF membrane, the floor of the prepared cavities was lined with type I glass ionomer cement (GIC) (Fuji I, GC Tokyo, Japan) (Fig. 1K). After ensuring that GIC was set, the cavity walls were etched with $37 \%$ orthophosphoric acid (Tetric Etch, Ivoclar Vivadent) for 20 seconds, rinsed and dried followed by application of bonding agent (Tetric Bond, Ivoclar Vivadent), and cured for 20 seconds in a light-curing unit. This was followed by restoration of the cavities with incremental addition and curing of light cure composite (Tetric N Ceram, Ivoclar Vivadent) for 20 seconds and the final increment was cured for 40 seconds (Fig. 1L). The restorations were assessed for the presence of premature contacts and visible marginal defects. The restoration was finished and polished with composite finishing kit (Shofu, Kyoto, Japan).

\section{Case 2}

\section{History}

A 22-year-old male patient reported to the department with a chief complaint of pain in right lower back teeth for the past 2 months. He gave a history of mild pain, which was stimulated by cold and sweet food, which lasted for a few seconds and subsided on the cessation of stimuli.

\section{Clinical Examination}

Intraoral examination revealed deep dentinal caries involving disto-occlusal surfaces in 46 (Fig. 2A). The tooth was not tender on percussion.

\section{Investigations}

Vitality testing using EPT and cold test (Endo-Ice, COLTENE) showed a positive response in 46 .

Radiographic findings revealed radiolucency in the distoocclusal aspect approximating pulp in relation to 46 , with no evidence of periodontal or periapical involvement (Fig. 2B).

\section{Provisional Diagnosis}

Reversible pulpitis in 46.

\section{Treatment Plan}

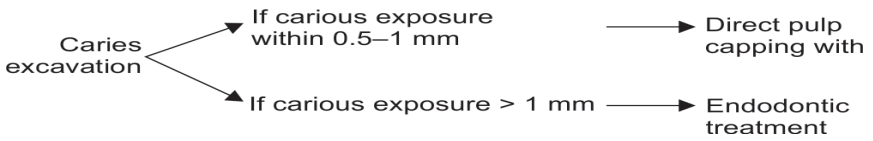

\section{Clinical Procedure}

Direct pulp-capping procedure: The caries excavation procedure was carried out as described in Case 1 (Fig. 2C).

PRF preparation: Platelet-rich fibrin was prepared after the operative procedure just before its placement in the cavity as a pulp-capping agent in accordance with the protocol developed by Choukroun et al. Ten milliliters of venous blood were drawn from the patient's antecubital vein and transferred into a sterile test tube without an anticoagulant. These tubes were immediately centrifuged in the Remi centrifugation machine at 2,700 rpm for 12 minutes (Fig. 2D). At the end of the process, a structured fibrin clot formed in the middle of the tube, just between acellular plasma (PPP) at the top
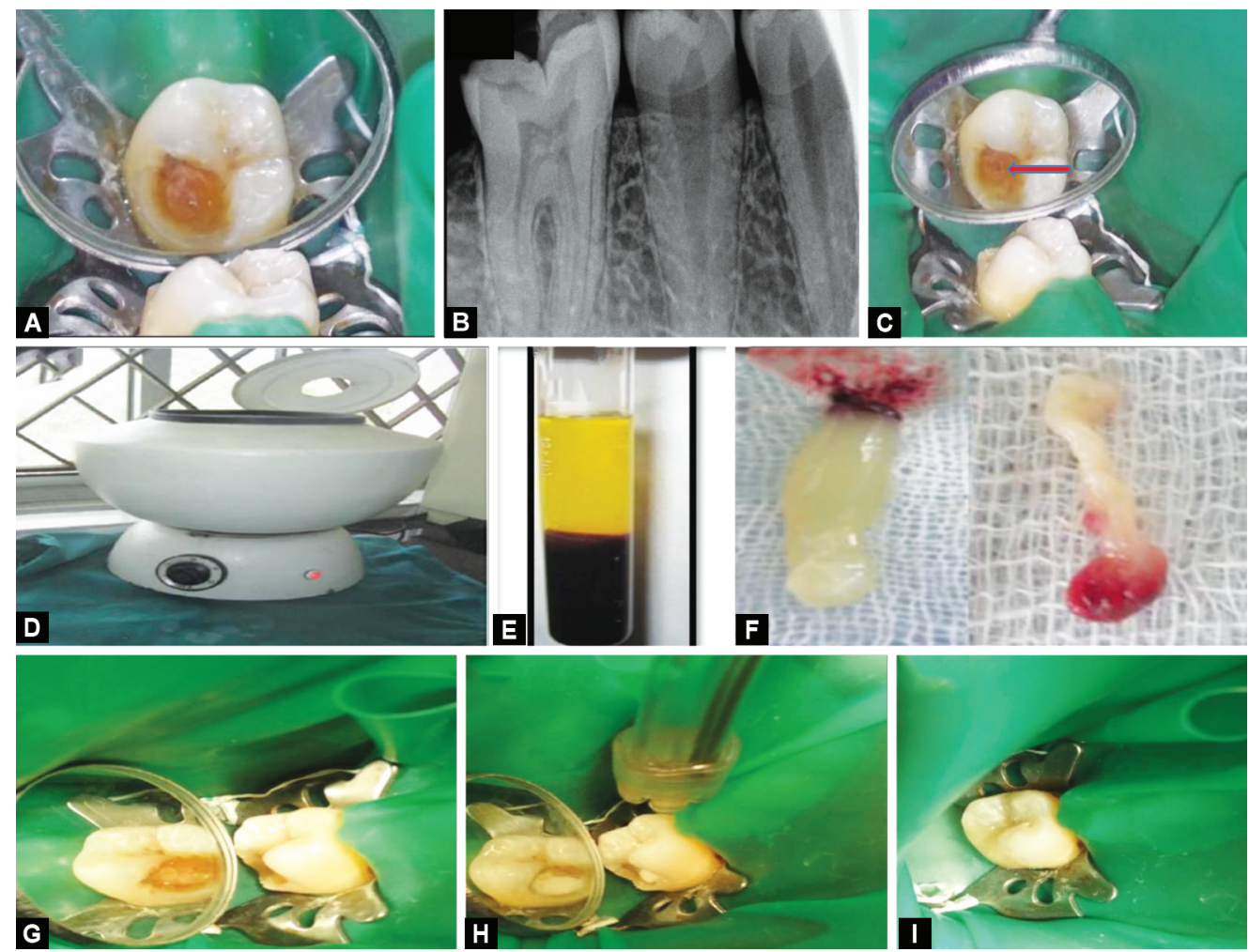

Figs 2A to I: (A) Preoperative image; (B) Preoperative radiograph; (C) Pinpoint exposure; (D and E) PRF preparation in Remi centrifugation machine; (F) PRF gel and membrane; (G) PRF placement; (H) GIC liner; (I) Light-cure composite restoration 
and the red corpuscles at the bottom (Fig. 2E). Platelet-rich fibrin was separated from red corpuscles base using sterile tweezers just after removal of PPP and then transferred into a sterile dappen dish. The PRF clot was compressed gently between moist sterile cotton gauze to remove the serum and to procure the PRF membrane (Fig. 2F). The PRF membrane was then carried to the exposure and positioned with the tine of the sterile explorer (Fig. 2G).

Restorative procedure: The restorative procedure was carried out as described in Case-1 (Figs 2H and I).

\section{Case 3}

History

A 32-year-old female patient reported to the department with a chief complaint of pain in right lower back teeth for the past 1 month. She gave a history of mild pain, which was stimulated by cold and sweet food, which lasted for a few seconds and subsided on the cessation of stimuli.

\section{Clinical Examination}

Intraoral examination revealed deep dentinal caries involving disto-occlusal surface in 46 (Fig. 3A). The tooth was not tender on percussion.

\section{Investigations}

Vitality testing using EPT and cold test (Endo-Ice, COLTENE) showed a positive response in 46 .
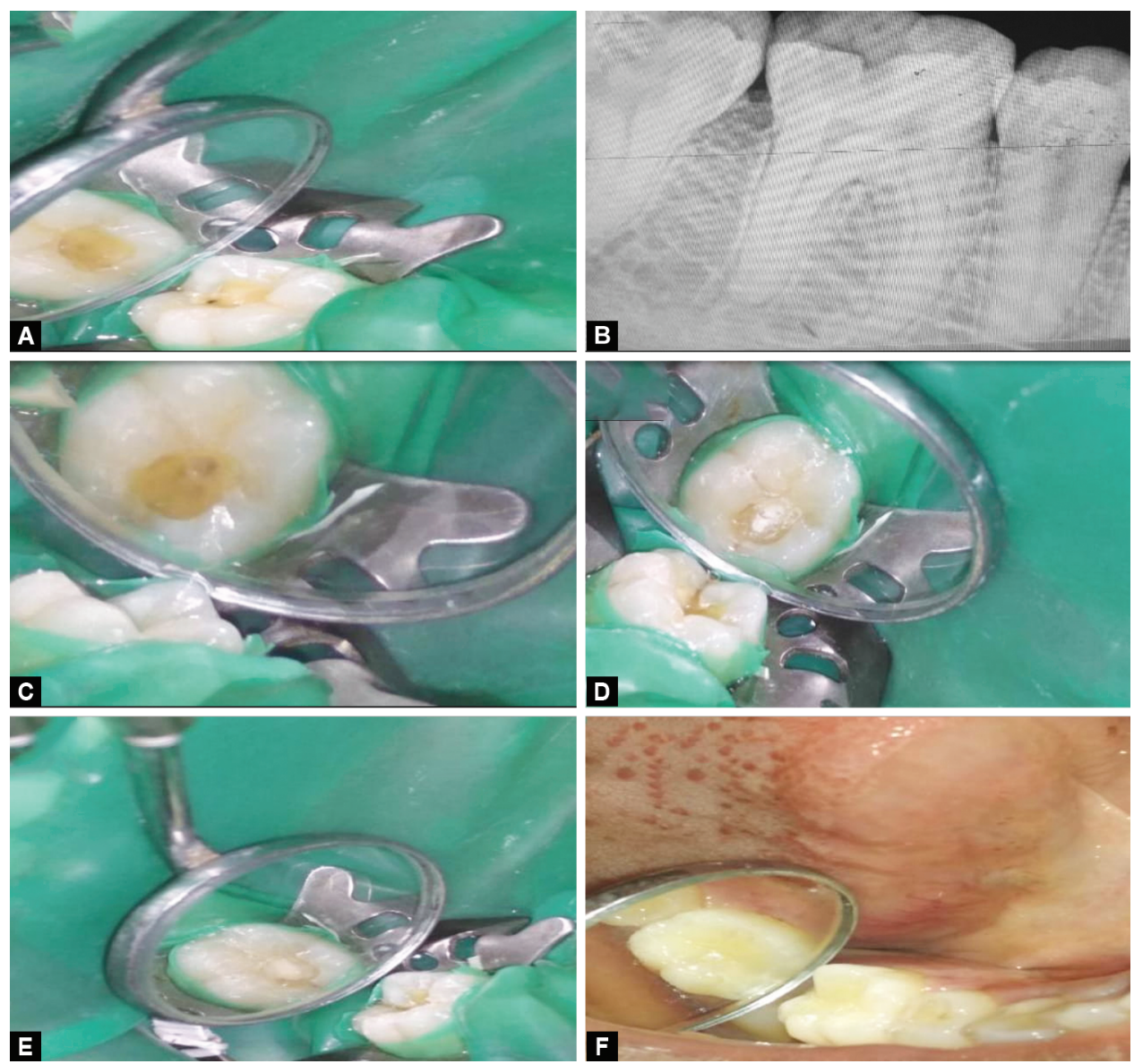

Figs 3A to F: (A) Preoperative image; (B) Preoperative radiograph; (C) Pinpoint exposure; (D) MTA placement; (E) GIC liner; (F) Composite restoration 

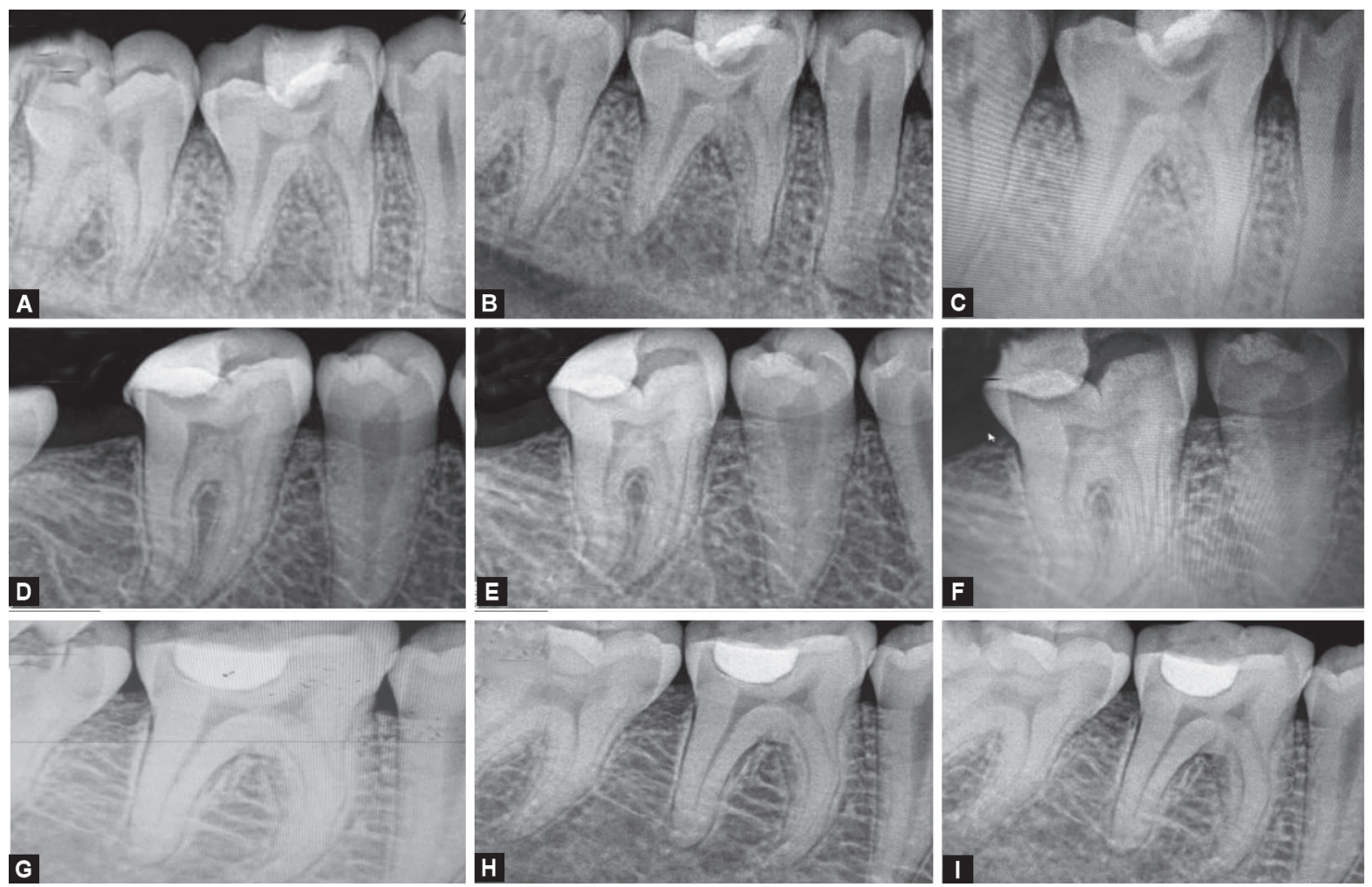

Figs 4A to I: (A-C) Direct pulp capping with CGF: (A) 3-month follow-up; (B) 6-month follow-up; (C) 12-month follow-up. (D-F) Direct pulp capping with PRF: (D) 3-month follow-up, (E) 6-month follow-up; (F) 12-month follow-up. (G-I) Direct pulp capping with MTA: (G) 3-month follow-up, (H) 6-month follow-up; (I) 12-month follow-up

\section{Postoperative Follow-up Clinical Follow-up}

Patients were reviewed after 3,6, and 12 months. The pulp vitality using cold and electric pulp testing showed a positive response, periodontal probing depth was normal, there was no mobility, no pain, and tenderness to percussion with respect to all the three cases.

\section{Radiographic Follow-up}

The pulp capped teeth were radiographically evaluated using Digital radiography (CS Image Software) with XCP film positioning device and standard exposure modes of $100 \mathrm{~ms}, 4 \mathrm{~mA}$, and $60 \mathrm{kVP}$.

There was no signs of periodontal space widening or periapical pathology in any of the three cases, at the 3-, 6-, and 12-month follow-up (Figs 4 A to I).

\section{Discussion}

All the three materials used in these cases are based on the biological approach of vital pulp therapy, which explores the molecular and cellular mechanisms behind pulp tissue regeneration and identifies a biological strategy for the treatment of clinical exposures. ${ }^{5}$ In the category of newer synthetic materials, MTA has shown promising clinical outcomes. A study showed that a high $\mathrm{pH}$ of 12.5 created in the area adjacent to the MTA remains high for at least 8 weeks and this high pH of MTA during setting has been found to affect cell growth and exert a cytotoxic effect on both macrophages and fibroblasts. ${ }^{6}$
Therefore, it is important to develop biocompatible treatments directed at maintaining pulp vitality and increasing tooth longevity, like the platelet concentrates. Though MTA is proved to induce dentin bridge formation by inducing biochemical pathways, platelet concentrates provide the necessary bioactive substances in ready-made form and they circumvent the natural biological process of induction. ${ }^{\text {? }}$

Platelet-rich fibrin is a second-generation platelet concentrate, developed by Choukroun et al. in 2001. Huang et al. investigated effect of PRF on cultured primary dental pulp cells and they concluded that PRF causes proliferation of human dental pulp cells and increase the protein expression of osteoprotegerin activity leading to the odontoblastic differentiation and mineralization process. ${ }^{8}$

Concentrated growth factor is an advanced second-generation platelet concentrate, developed by Sacco in 2006. Concentrated growth factor appears to contain more abundant cytokines and was substantially studied in bone regeneration, but there has been little research performed to substantiate its role as a pulp-capping agent.

Platelet concentrates can increase dental pulpal cells (DPC) proliferation and differentiation, suggesting potential applications of these as a biological molecule to promote the regeneration of lost or injured dental pulp tissues and stimulate reparative dentinogenesis.

\section{Conclusion}

Platelet concentrates like PRF and CGF were found to have clinical efficacy that was comparable to MTA. Thus, they could be considered as a promising alternative to MTA as direct pulpcapping agents. 


\section{Clinical Significance}

The promising results of platelet concentrates as direct pulpcapping agents opens a new avenue for vital pulp therapy procedures.

\section{References}

1. Glossary of endodontic terms. American Association of Endodontists. 7th ed., Chicago (IL) 2003.

2. Bogen G, Kim JS, Bakland LK. Direct pulp capping with mineral trioxide aggregate: an observational study. J Am Dent Assoc 2008;139(3):305-315. DOI: 10.14219/jada.archive.2008.0160.

3. Parirokh M, Torabinejad M. Mineral trioxide aggregate: a comprehensive literature review-part III: clinical applications, drawbacks, and mechanism of action. J Endod 2010;36(3):400-413. DOI: 10.1016/j.joen.2009.09.009.
4. Zhang $M$, Jiang $F$, Zhang $X$, et al. The effects of platelet derived growth factor-BB on human dental pulp stem cells mediated dentin-pulp complex regeneration. Stem Cells Transl Med 2017;6(12):2126-2134. DOI: 10.1002/sctm.17-0033.

5. Keswani D, Pandey RK, Ansari A, et al. Comparative evaluation of platelet-rich fibrin and mineral trioxide aggregate as pulpotomy agents in permanent teeth with incomplete root development: a randomized controlled trial. J Endod 2014;40(5):599-605. DOI: 10.1016/j.joen.2014.01.009.

6. Fridland M, Rosado R. MTA solubility: a long term study. J Endod 2005;31(5):376-379. DOI: 10.1097/01.DON.0000140566.97319.3e.

7. Tabatabayi MH, Tavakoli A, Ameghani BA. Regenerative property of PRF used as capping material in pulpotomy in dogs. Biomed Res 2017;28(10).

8. Huang FM, Yang SF, Zhao JH, et al. Platelet-rich fibrin increases proliferation and differentiation of human dental pulp cells. J Endod 2010;36(10):1628-1632. DOI: 10.1016/j.joen.2010.07.004. 